\title{
Nuclear Factor of Activated T Cells and Cytokines Gene Expression of the T Cells in AIDS Patients with Immune Reconstitution Inflammatory Syndrome during Highly Active Antiretroviral Therapy
}

\author{
Jia Sun, ${ }^{1}$ Heling Chen, ${ }^{1}$ Yirui Xie, ${ }^{1}$ Junwei Su, ${ }^{1}$ Ying Huang, ${ }^{1}$ Lijun Xu, \\ Michael Yin, ${ }^{2}$ Qihui Zhou, ${ }^{1}$ and Biao Zhu ${ }^{1}$ \\ ${ }^{1}$ State Key Laboratory for Diagnosis and Treatment of Infectious Diseases, Collaborative Innovation Center for Diagnosis and \\ Treatment of Infectious Diseases, The First Affiliated Hospital, School of Medicine, Zhejiang University, Hangzhou 310003, China \\ ${ }^{2}$ Division of Infectious Diseases, Columbia University Medical Center, New York, NY 10032, USA \\ Correspondence should be addressed to Biao Zhu; zhubiao1207@zju.edu.cn
}

Received 27 October 2016; Accepted 24 January 2017; Published 20 February 2017

Academic Editor: Vera L. Petricevich

Copyright (C) 2017 Jia Sun et al. This is an open access article distributed under the Creative Commons Attribution License, which permits unrestricted use, distribution, and reproduction in any medium, provided the original work is properly cited.

\begin{abstract}
Background. The etiology of immune reconstitution inflammatory syndrome (IRIS) in AIDS patients after the initiation of HAART remains unknown. Several researches indicated that the development of IRIS is associated with the production and variation of cytokines, whose gene expression are closely related to the $\mathrm{Ca}_{2}^{+} / \mathrm{CN}$-nuclear factor of activated T cells (NFAT) pathway. Methods. We studied the expression of NFAT isoforms and their major target cytokines genes in peripheral blood CD $3^{+} \mathrm{T}$ cells of subjects through fluorescence quantitative PCR and explored the expression changes of these genes before and after HAART. Results. After the initiation of HARRT, NFAT1, IL-6, and IL-8 gene expression showed a reversal trend in the CD $3^{+} \mathrm{T}$ cells of the IRIS group and changed from low expression before HARRT to high expression after HARRT. In particular, the relative gene expression of NFAT1 was markedly higher compared with the other three isoforms. The IRIS group also showed higher NFAT4, NFAT2, NFAT1, IL-1 $\beta$, IL-10, IL-2, IL-18, and TNF- $\alpha$ gene expression than the non-IRIS group. Conclusion. This study suggested that high expression levels of IL-2, IL-6, IL- 8 , TNF- $\alpha$, IL-1 $\beta$, IL-10, IL-12, and IL-18 can predict the risk of IRIS. The increased expression of NFAT1 and NFAT4 may promote the expression of cytokines, such as IL-6, IL-8, and TNF- $\alpha$, which may promote the occurrence of IRIS.
\end{abstract}

\section{Introduction}

Highly active antiretroviral therapy (HAART) has substantially reduced the incidence and mortality of HIV-related diseases in AIDS patients. However, $8-40 \%$ of patients still have excessive inflammatory responses to various infectious or noninfectious pathogens, mostly within six months of HAART, leading to increased clinical symptoms. These patients present with aggregated opportunistic infections that have been effectively treated or with emerging infections, which is called immune reconstitution inflammatory syndrome (IRIS) [1-5]. IRIS is associated with various infectious diseases, and tuberculosis-related IRIS constitutes the highest incidence of IRIS at $8-43 \%$; the incidence is even higher in developed countries (17-43\%) [6]. Other infections associated with IRIS include Mycobacterium avium infection, cryptococcosis, pneumocystis pneumonia, and cytomegalovirus retinitis; noninfectious diseases include various autoimmune diseases such as Graves' disease and Kaposi's sarcoma [7].

IRIS is likely to occur in patients who have high viral loads and low CD4 cardinality $(<50 / \mu \mathrm{L})$ before HAART, with a rapid decline in the viral load and a fast increase in CD4 counts after HAART. Additionally, IRIS easily occurs in patients who have a short interval between the treatment of opportunistic infections and the start of HAART. Moreover, IRIS has been reported to easily occur in patients with low baseline hemoglobin but high C-reactive protein and plasma D-dimer levels [5-10]. However, the pathogenesis of IRIS 
remains unclear, and there are great differences among the various types of IRIS. It has been reported that tuberculosisassociated IRIS (TB-IRIS) is associated with interleukin-4 (IL-4), IL-6, IL-7, IL-12p40, interferon- $\gamma$ (IFN- $\gamma$ ), and tumor necrosis factor- $\alpha$ (TNF- $\alpha)$; an elevation in IL-12 can increase the incidence of cytomegalovirus-associated IRIS (CMVIRIS), whereas IFN- $\gamma$, IL-12p40, IL-6, IL-4, IL-17, and TNF$\alpha$ are relevant to Cryptococcus neoformans-associated IRIS (C. neoformans-IRIS) [11-13]. The nuclear factor of activated $\mathrm{T}$ cells (NFAT) is expressed on the promoter/enhancer of multiple genes mentioned above, including IL-2, IL-3, IL-4, IL-5, IL-6, IL-8, IL-10, IL-13, IFN- $\gamma$, TNF- $\alpha$, and granulocytemacrophage colony-stimulating factor (GM-CSF); NFAT regulates the expression of its target genes mainly through the $\mathrm{Ca}^{2+} / \mathrm{CN}-\mathrm{NFAT}$ pathway [14-16].

To further elucidate the pathogenesis of IRIS, we analyzed gene expression changes of NFAT1-NFAT4 and the major target genes IL-2, IL-6, IL-8, TNF- $\alpha$, IL- $1 \beta$, IL-10, and IL-12 in peripheral blood $\mathrm{CD}^{+} \mathrm{T}$ cells before and during the course of IRIS.

\section{Materials and Methods}

2.1. Study Population and Baseline Profile. This study enrolled 63 AIDS patients at the Infectious Diseases Center of the First Affiliated Hospital, Zhejiang University School of Medicine (Hangzhou, Zhejiang Province, China), from March 2003 to December 2014, of which 19 subjects have been diagnosed with IRIS at the time they entered the study. Of the remaining 44 patients, 5 have initiated HAART for 2 weeks when they agreed to participate in the study, and 39 subjects have not. Under observation for as long as six months, 11 subjects had IRIS and 33 did not. Above all, there were 30 patients with IRIS (IRIS group: 28 males and 2 females) and 33 patients without IRIS (non-IRIS group: 30 males and 3 females). The IRIS group included 13 cases with TB-IRIS, five cases with CMV-IRIS, three cases with C. neoformansIRIS, five cases with varicella zoster virus-associated IRIS, two cases with pneumocystis pneumonia-associated IRIS, one case with cytomegalovirus/tuberculosis coinfected IRIS, and one case with Penicillium marneffei-associated IRIS. IRIS was diagnosed according to the criteria of the International Network for the Study of HIV-Associated IRIS. The baseline conditions of the subjects are detailed in Table 1. This study was approved by the hospital ethics committee, and each patient who was enrolled had signed an informed consent form.

2.2. Specimen Collection and Processing. Whole blood specimens $(5-8 \mathrm{~mL}$ each) were collected from the all subjects at the initiation and 3 months after starting HAART. Moreover, for the IRIS group, blood specimens were collected at the diagnosis time point of IRIS and 1 month after HAART for the non-IRIS group. The specimens were anticoagulated with EDTA. Plasma and PBMCs were isolated within 10 hours of collection. The PBMCs were stored in Zenoaq's CELLBANKER cryopreservation medium at $-80^{\circ} \mathrm{C}$. We examined gene expression changes in the IRIS and non-IRIS groups before and 1 month after HAART.

\subsection{Detection of NFAT and the Expression of Its Target Genes in T Cells}

2.3.1. Magnetic Bead Separation of $\mathrm{CD}^{+} \mathrm{T}$ Cells and RNA Extraction of Samples. The PBMCs were thawed in a warm bath at $37^{\circ} \mathrm{C}$ and centrifuged at $300 \mathrm{~g} / \mathrm{min}$ for $5 \mathrm{~min}$. The supernatant was aspirated, and the $\mathrm{CD}^{+} \mathrm{T}$ cells were isolated using CD3 human MicroBeads (Miltenyi Biotec, Bergisch Gladbach, Germany) by strictly following the manufacturer's instructions. The isolated $\mathrm{CD}^{+} \mathrm{T}$ cells were added to $1 \mathrm{~mL}$ of TRIzol reagent (Invitrogen, Life Technologies, Carlsbad, CA, USA), and total RNA extraction was performed according to the manufacturer's instructions. The extracted RNA was dissolved with $20 \mu \mathrm{L}$ of DEPC water before the RNA concentration measurement.

2.3.2. cDNA Synthesis. The cDNA synthesis reactions were prepared on ice using a PrimeScript RT reagent kit (Takara, Dalian, China). The $40 \mu \mathrm{L}$ reaction contained $8 \mu \mathrm{L}$ of $5 \mathrm{x}$ PrimeScript Buffer (for real time), $2 \mu \mathrm{L}$ of PrimeScriptRT Enzyme Mix I, $2 \mu \mathrm{L}$ of Oligo dT Primer $(50 \mu \mathrm{M}), 2 \mu \mathrm{L}$ of random 6 mers $(100 \mu \mathrm{M}), 500 \mathrm{ng}$ of total RNA, and up to $40 \mu \mathrm{L}$ of $\mathrm{RNase}$-free $\mathrm{dH}_{2} \mathrm{O}$. The reaction conditions were as follows: $37^{\circ} \mathrm{C}$ for $45 \mathrm{~min}, 85^{\circ} \mathrm{C}$ for $5 \mathrm{sec}$, and $4^{\circ} \mathrm{C}$ for 1 hour. The synthesized cDNA was stored at $-20^{\circ} \mathrm{C}$.

2.3.3. Fluorescence Quantitative RT-PCR. We performed qPCR reactions using $\mathrm{iQ}^{6}$ SYBR Green Supermix (Bio-Rad, Hercules, CA, USA). The reaction system contained $12.5 \mu \mathrm{L}$ of Bio-Rad iQ SYBR Green Supermix, $1 \mu \mathrm{L}$ of sense primer (50 pg), $1 \mu \mathrm{L}$ of antisense primer ( $5 \mathrm{pg}), 1 \mu \mathrm{L}$ of cDNA, and $9.5 \mu \mathrm{L}$ of DEPC $\mathrm{H}_{2} \mathrm{O}$, resulting in a total volume of $25 \mu \mathrm{L}$. The reaction conditions were as follows: $95^{\circ} \mathrm{C}$ for $3 \mathrm{~min}$, followed by 45 cycles of $95^{\circ} \mathrm{C}$ for $15 \mathrm{sec}, 60^{\circ} \mathrm{C}$ for $30 \mathrm{sec}$, and $72^{\circ} \mathrm{C}$ for $30 \mathrm{sec}$. A melting curve analysis was performed using the following conditions: $65^{\circ} \mathrm{C}$ to $95^{\circ} \mathrm{C}$ for $5 \mathrm{~s}$. The primer sequences are shown in Table 2. The primers were synthesized by Sangon (Shanghai, China). The PCR reactions were performed on a quantitative PCR machine (Bio-Rad).

\section{Statistical Methods}

All of the data were analyzed using IBM SPSS Statistics 19 (IBM SPSS, Somers, NY, USA) unless otherwise stated. We first performed a one-sample K-S test to examine the normal distribution of the data. Then, a $T$-test was used to analyze the normally distributed data, and the Mann-Whitney $U$ test was used to analyze the nonnormally distributed data. Differences were considered statistically significant at $p<0.05$. All of the graphs were generated using GraphPad Prism 5 (GraphPad Software, Inc., San Diego, CA, USA). We compared previous gene expression changes to 1 month after HAART in the IRIS and non-IRIS groups, respectively. Meanwhile, we compare IRIS group to non-IRIS group at the time before HAART and 1 month after HAART separately by using the fold difference $\left(2^{-\Delta \Delta \mathrm{CT}}\right)$ method, with human GAPDH as an internal reference. The Delta-Delta-Threshold cycle $(\Delta \Delta \mathrm{CT})$ equation was used to compare the expressions of NFAT and cytokine genes in IRIS and non-IRIS patients before and after 
TABLE 1: General information of the 63 AIDS patients enrolled in this study.

\begin{tabular}{|c|c|c|c|}
\hline & $\begin{array}{c}\text { IRIS (30 } \\
\text { cases) }\end{array}$ & $\begin{array}{c}\text { Non-IRIS (33 } \\
\text { cases) }\end{array}$ & $p$ \\
\hline \multicolumn{4}{|l|}{ Gender } \\
\hline Male & 28 & 30 & $>0.05$ \\
\hline Female & 2 & 3 & $>0.05$ \\
\hline Average age & 40.5 & 35.8 & $>0.05$ \\
\hline \multicolumn{4}{|l|}{$\mathrm{CD}^{+} \mathrm{T}\left(\mathrm{cells} / \mathrm{mm}^{3}\right)$} \\
\hline Pretreatment & 45 & 150 & $<0.001$ \\
\hline 1-Month treatment & 135 & 295 & $>0.05$ \\
\hline \multicolumn{4}{|l|}{ HIV-RNA (log 10 copies $/ m L)$} \\
\hline Pretreatment & 5.52 & 5.627 & $>0.05$ \\
\hline 1-Month treatment & 4.22 & 5.36 & $>0.05$ \\
\hline \multicolumn{4}{|l|}{ Hemoglobin $(g / d L)$} \\
\hline $\begin{array}{l}\text { Pretreatment } \\
\text { Mean duration of IRIS (days) }\end{array}$ & $\begin{array}{c}11.5 \\
31\end{array}$ & 13.6 & $>0.05$ \\
\hline \multicolumn{4}{|l|}{ Therapeutic strategy } \\
\hline 2NRTI + NNRTI & 27 & 30 & $>0.05$ \\
\hline 2NRTI + PI & 3 & 3 & $>0.05$ \\
\hline
\end{tabular}

NRTI: nucleoside reverse transcriptase inhibitors; NNRTI: nonnucleoside reverse transcriptase inhibitors; PI: protease inhibitor.

TABLE 2: Primer sequences.

\begin{tabular}{|c|c|c|}
\hline Gene & Primer sequence & Product length $(\mathrm{bp})$ \\
\hline \multirow{2}{*}{ IL-2 } & F: TGCATTGCACTAAGTCTTGCAC & \multirow{2}{*}{194} \\
\hline & R: AGTTCTGTGGCCTTCTTGGG & \\
\hline \multirow{2}{*}{ TNF- $\alpha$} & F: CCCTGGTATGAGCCCATCTAT & \multirow{2}{*}{298} \\
\hline & R: CGAAGTGGTGGTCTTGTTGC & \\
\hline \multirow{2}{*}{ IL-8 } & F: TGAATGGGTTTGCTAGAATGTG & \multirow{2}{*}{223} \\
\hline & R: CTGTGAGGTAAGATGGTGGCTAA & \\
\hline \multirow{2}{*}{ IL-6 } & F: AGTAGTGAGGAACAAGCCAGAG & \multirow{2}{*}{240} \\
\hline & R: ATGCTACATTTGCCGAAGAG & \\
\hline \multirow{2}{*}{ IL-10 } & F: GCCCCTTGAGAAACCTTATTGT & \multirow{2}{*}{115} \\
\hline & R: GGCTTCTTTCTAAATCGTTCACAG & \\
\hline \multirow{2}{*}{ IL-1 $\beta$} & F: GCACCTCTCAAGCAGAAAACA & \multirow{2}{*}{207} \\
\hline & R: ACAACAGGAAAGTCCAGGCTAT & \\
\hline \multirow{2}{*}{ IL-18 } & F: GCTGAAGATGATGAAAACCTGGA & \multirow{2}{*}{118} \\
\hline & R: GAGGCCGATTTCCTTGGTCA & \\
\hline \multirow{2}{*}{ IL-12A } & F: TTCACСАСТCССАAААССТGC & \multirow{2}{*}{226} \\
\hline & R: GAGGCCAGGCAACTCCCATTAG & \\
\hline \multirow{2}{*}{ NFAT1 } & F: AAGAGCCAGCCCAACATGC & \multirow{2}{*}{107} \\
\hline & R: CGTTTTCTCTTCCCATTGATGAC & \\
\hline \multirow{2}{*}{ NFAT2 } & F: CTGTGCAAGCCGAATTCTCTGG & \multirow{2}{*}{78} \\
\hline & R: ACTGACGTGAACGGGGCTGG & \\
\hline \multirow{2}{*}{ NFAT4 } & F: GCGGCCTGCAGATCTTGAGC & \multirow{2}{*}{102} \\
\hline & R: TGATGTGGTAAGCAAAGTGGTGTGGT & \\
\hline \multirow{2}{*}{ GAPDH } & F: GGCCTCCAAGGAGTAAGACC & \multirow{2}{*}{147} \\
\hline & R: AGGGGTCTACATGGAAACTG & \\
\hline
\end{tabular}


TABLE 3: Fold change expression and quantitative PCR results of NFAT and cytokines genes.

\begin{tabular}{lcccc}
\hline & Fold difference 1 & Fold difference 2 & Fold difference 3 & Fold difference 4 \\
\hline NFAT4 & 0.661 & 0.969 & 2.017 & 2.957 \\
NFAT2 & 0.473 & 0.983 & 1.661 & 3.452 \\
NFAT1 & 0.493 & 3.002 & 0.265 & 1.612 \\
IL-2 & 0.428 & 0.329 & 2.223 & 1.707 \\
IL-8 & 0.298 & 1.210 & 0.829 & 3.368 \\
IL-1 $\beta$ & 0.629 & 1.027 & 1.836 & 3.000 \\
IL-6 & 0.329 & 1.629 & 0.687 & 3.406 \\
IL-10 & 0.541 & 0.451 & 2.674 & 2.229 \\
TNF- $\alpha$ & 1.013 & 1.335 & 1.533 & 2.050 \\
IL-18 & 0.512 & 0.250 & 2.536 & 1.239 \\
IL-12 & 0.301 & 0.111 & 2.525 & 0.934 \\
\hline
\end{tabular}

Fold difference $=[2]^{-\Delta \Delta C T}$.

$\Delta \Delta \mathrm{CT} 1=$ [average $\Delta \mathrm{CT}$ value of non-IRIS group after HAART - average $\Delta \mathrm{CT}$ value of non-IRIS group before HAART].

$\Delta \Delta \mathrm{CT} 2=$ [average $\Delta \mathrm{CT}$ value of IRIS group after HAART - average $\Delta \mathrm{CT}$ value of IRIS group before HAART].

$\Delta \Delta \mathrm{CT} 3=$ [average $\Delta \mathrm{CT}$ value of IRIS group before HAART - average $\Delta \mathrm{CT}$ value of non-IRIS group before HAART].

$\Delta \Delta \mathrm{CT} 4=$ [average $\Delta \mathrm{CT}$ value of IRIS group after HAART - average $\Delta \mathrm{CT}$ value of non-IRIS group after HAART].

HAART in terms of fold difference according to the following formulas:

$\Delta \mathrm{CT}=\mathrm{CT}$ value of targeted gene $-\mathrm{CT}$ value of GAPDH.

$\Delta \Delta \mathrm{CT}=$ [average $\Delta \mathrm{CT}$ value of the IRIS group average $\Delta C T$ value of the non-IRIS group] or [average $\triangle \mathrm{CT}$ value of patients before HAART - average $\Delta \mathrm{CT}$ value of patients after HAART].

Fold difference $=[2]^{-\Delta \Delta C T}$.

\section{Results}

4.1. NFAT and Cytokine Gene Relative Expression Levels Compared before and after HAART in the IRIS and NonIRIS Groups of AIDS Patients. A comparative analysis of the relative gene expression by fold difference before and after HAART revealed that the IRIS group had higher NFAT1, IL6 , IL-8, and TNF- $\alpha$ expression in $\mathrm{CD}^{+}$T cells after receiving HAART for 1 month. The gene expression increase was the most obvious for NFAT1, whose expression after HAART was 3.058-fold greater than that before HAART. However, gene expression of IL-2, IL-10, and IL-18 was lower after HAART. The differences of gene expressions of NFAT4, NFAT2, and IL- $1 \beta$ were not obvious after initiation of HAART (fold difference: $0.969,0.981$, and 1.03 , resp.). The non-IRIS group showed lower NFAT4, NFAT2, NFAT1, IL-1 $\beta$, IL-6, IL-8, IL-10, IL-2, and IL-18 gene expressions after HAART than before HAART. The gene expression of TNF- $\alpha$ after HAART changed little, and it was 1.03-fold higher than that before HAART (Figure 1 and Table 3 ).

4.2. NFAT and Cytokine Gene Relative Expression Levels Comparing the IRIS to Non-IRIS Groups of AIDS Patients before and after HAART. We compared the relative gene expression levels of NFAT and cytokines between the IRIS and non-IRIS groups before HAART. NFAT1 was expressed at significantly lower levels than the other three NFAT isoforms in the $\mathrm{CD}^{+} \mathrm{T}$ cells of the IRIS group. The IRIS group showed lower IL-6 and IL-8 expression levels than the nonIRIS group, whereas other genes including NFAT4, NFAT3, NFAT2, IL-1 $\beta$, IL-10, IL-2, IL-18, and TNF- $\alpha$ were expressed at higher levels in the IRIS group than in the non-IRIS group. After 1 month of HAART, NFAT1, IL-6, and IL-8 expressions showed a reversal trend and changed from low expression levels before HAART to high expression levels after HAART. The relative expressions of NFAT1, IL- 6 , and IL- 8 genes in the IRIS group were 1.61-, 3.406-, and 3.368-fold greater than those in the non-IRIS group, respectively; other genes like NFAT4, NFAT2, IL-1 $\beta$, IL-2, IL-10, TNF- $\alpha$, and IL-18 were also expressed at higher levels compared with those in the non-IRIS group. However, the relative expressions of IL-10, IL-2, and IL-18 showed a larger decreasing amount than those in the non-IRIS group (Figure 2 and Table 3 ).

\section{Discussion}

IRIS easily occurs in patients who have a short interval between the treatment of opportunistic infections and the start of HAART [5]. As a result, the occurrence of IRIS was related with residual antigen burden. Taken the active tuberculosis as an example, before antituberculosis therapy, there existed high level of TB antigen, so the first step for TB AIDS patients was anti-TB therapy and then HAART, which was similar in the therapy of CMV AIDS. Generally speaking, the development of IRIS associated with pathogen infection; therefore the major principle of IRIS treatment is the antipathogen therapy for a period of time before the initiation of HAART, through which it can not only effectively decrease the antigen burden but also cut down the scale of residual antigen. Meanwhile, to reduce the occurrence of IRIS, methods like T-SPOT, CMV-DNA, and VZV-DNA are widely used to measure the antigen burden and residual antigen in clinic. 


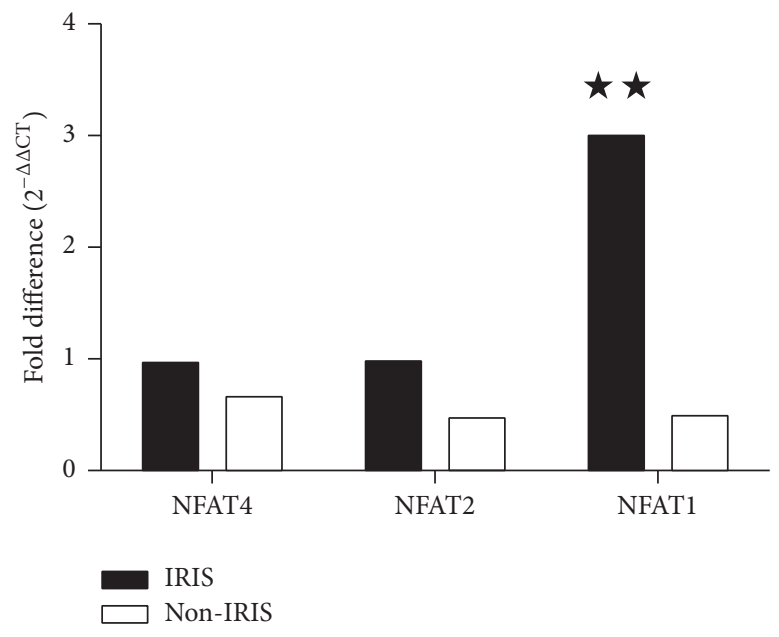

(a)

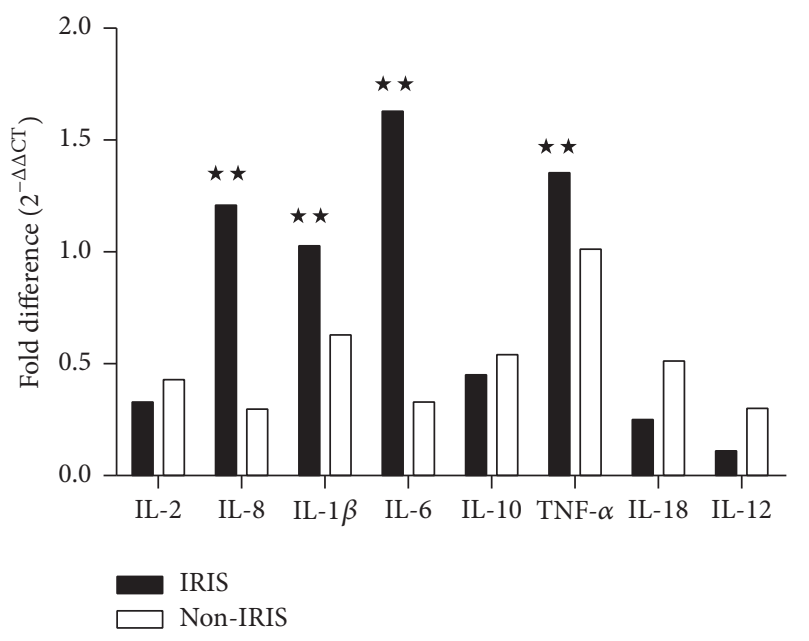

(b)

FIGURE 1: NFAT and cytokine gene relative expression levels compared before and after HAART in the immune reconstitution inflammatory syndrome (IRIS) and non-IRIS groups of AIDS patients. (a) NFAT expression in IRIS groups before and after HAART; NFAT1 expression from low to high after HAART; the relative expression of mRNA for NFAT1 gene to GAPDH after HAART was 3.002-fold higher as compared to that before HAART. (b) Cytokine gene expression in IRIS groups before and after HAART; IL- $1 \beta$, IL-6, IL-8, and TNF- $\alpha$ expression from low to high; TNF- $\alpha$ gene expression also increased in non-IRIS group after HAART; ${ }^{\star}$ the gene expressions after HAART were higher than those before treatment.

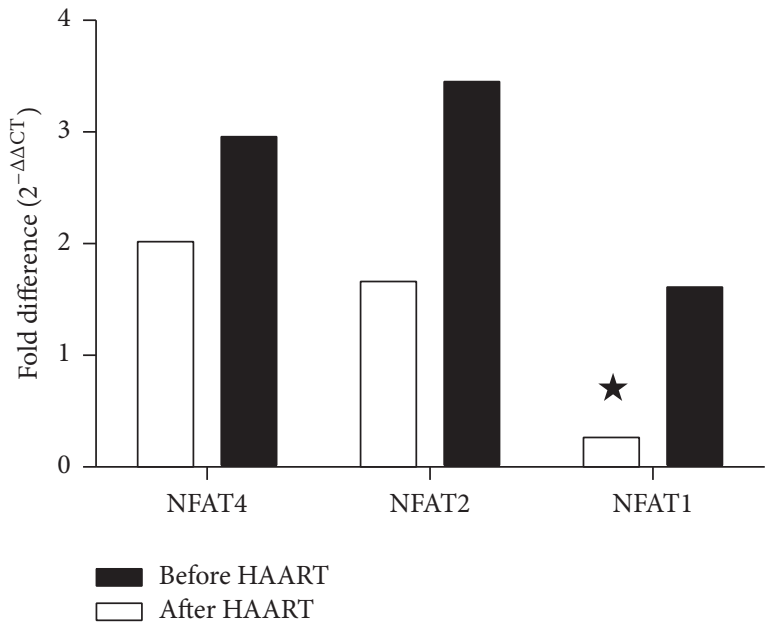

(a)

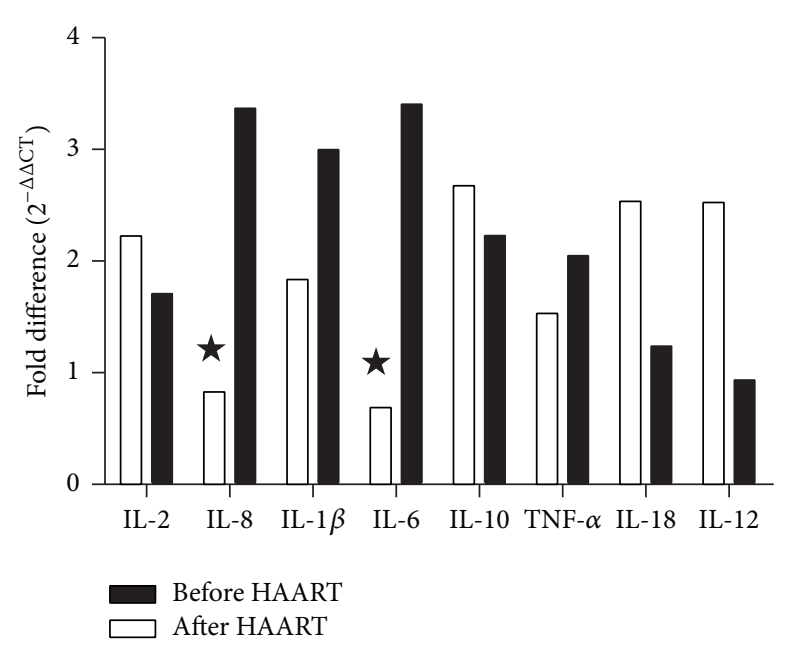

(b)

FIGURE 2: NFAT and cytokine gene relative expression levels comparing the IRIS to non-IRIS groups of AIDS patients before and after HAART. (a) NFAT expression in IRIS and non-IRIS groups before HAART. NFAT1 expression was obviously lower in IRIS group. (b) Cytokine gene expression in IRIS and non-IRIS groups before HAART. IL-6 and IL-8 expressions were obviously lower in IRIS group; ${ }^{\star}$ before HAART, the gene expressions in IRIS group were lower than those in non-IRIS group.

The pathogenesis of IRIS has not been clarified yet. Tadokera et al. found that IL-1 $\beta$, IL-5, IL-6, IL-10, IL-13, IL$17 \mathrm{~A}, \mathrm{IFN}-\gamma$, GM-CSF, and TNF levels are elevated in the cell culture of PBMCs from TB-IRIS patients after in vitro stimulation with inactivated Mycobacterium tuberculosis. This finding indicates that the release of cytokines promotes the occurrence of TB-IRIS [17]. The cytokine release syndrome (also known as the cytokine storm) can be observed in infectious or noninfectious diseases, such as graft-versus-host disease and acute respiratory distress syndrome [17]. Currently, calcineurin inhibitors including cyclosporine (CSA) and tacrolimus (FK506) are available to block the $\mathrm{Ca}^{2+}$ CN/NFAT pathway and suppress the function and spread of effector T cells. These agents have been extensively used in transplantation patients. Moreover, calcineurin inhibitors can inhibit the formation of IL-2, IL-4, and TNF- $\alpha$ [16]. 
Therefore, we speculate that the $\mathrm{Ca}^{2+}$-CN/NFAT pathway may play a role in the excessive immune response of AIDS patients receiving HAART.

We performed fluorescence quantitative PCR analysis to detect NFAT1-NFAT4, IL-2, IL-6, IL-8, TNF- $\alpha$, IL-1 $\beta$, IL-10, and IL-12 genes in the $\mathrm{CD}^{+} \mathrm{T}$ cells of IRIS and non-IRIS patients. The results showed that the relative gene expression of NFAT1, IL-6, IL-8, and TNF- $\alpha$ was obviously higher after 1 month of HAART than before HAART in the $\mathrm{CD}^{+} \mathrm{T}$ cells of IRIS patients and that the most significant gene expression increase was obtained for NFAT1. When the IRIS group was compared to the non-IRIS group, we found that, before HAART, IL- $1 \beta$, IL-10, and IL-2 expressions of $\mathrm{CD}^{+} \mathrm{T}$ cells were higher in the former than in the latter group; after 1 month of HAART, NFAT2, NFAT4, IL-6, IL-8, TNF- $\alpha$, IL-1 $\beta$, IL-10, and IL-2 expression levels were all higher in the former than in the latter group. IL-1 $\beta$, IL-10, and IL-2 expressions were reduced in both the IRIS and non-IRIS groups after HAART; the reduction was larger in the non-IRIS group than in the IRIS group. Maintaining relatively high expression levels of IL-1 $\beta$, IL-10, and IL-2 may play an important role in the development and progression of IRIS. The increase in NFAT1 expression in IRIS patients after 1 month of HAART likely plays an essential role in the occurrence of IRIS by contributing to IL-6, IL- 8 , and TNF- $\alpha$ expression,

NFAT can bind to the promoter/enhancer of various cytokine genes and can contribute to the expression of IL2, IL-3, IL-4, IL-5, IL-6, IL-8, IL-10, IL-13, IFN- $\gamma$, and TNF$\alpha$. During HAART for AIDS, we found NFAT expression changes in the T lymphocytes of the IRIS patients. Among four NFAT1 isoforms, NFAT1 and NFAT4 showed an increase in expression; the expression increase was more significant for NFAT1. These results provided a new idea for the treatment of IRIS: we can use selective inhibitors of iRNA or NFAT1 to block the CN/NFAT pathway and reduce IL-2, IL-3, IL-4, IL-5, IL-6, IL-8, IL-10, IL-13, IFN- $\gamma$, and TNF- $\alpha$ generation for the treatment of IRIS to reduce the occurrence of IRIS and improve the prognosis of IRIS patients in the clinic.

Taken powerful immunosuppressive action of calcineurin inhibitors, such as CSA and FK506, into consideration, we replace thalidomide, which is an anti-inflammatory, immunomodulatory, and antiangiogenic agent [18] and has already been effectively used in our recent case report of Mycobacterium avium complex- (MAC-) associated IRIS, whose cutaneous MAC lesions continue exacerbation despite antimycobacterial treatment. But rapid clinical remission occurred after adding thalidomide to the antituberculosis treatment. Furthermore, immunomodulatory effect of thalidomide on cytokine levels such as decreasing of TNF$\alpha$ and increasing of IL- 4 and IL- 6 was observed in the patient [19]. The limitation for this approach of blocking the CN/NFT pathway is that this inhibition could negatively affect immune responses activation when these patients have real/new infections, as the immune system is still under severely immune suppression. Anyway, the development of IRIS also indicated that these patients have an excellent effect in response to HAART. As it is a condition of immunoimbalance, under the written informed consents from all subjects, we are conducting a case-control study by using CN/NFT drugs from small doses in study group and observe the effects intensively.

\section{Consent}

Written consent was obtained from the patients.

\section{Competing Interests}

All authors declare that they have no potential competing interests.

\section{Acknowledgments}

This work was funded by National Special Research Program for Important Infectious Diseases (2013ZX10001004-002-003 and 2014ZX10001002-002-002).

\section{References}

[1] S. D. Lawn and R. Wood, "Immune reconstitution inflammatory syndrome," The Lancet Infectious Diseases, vol. 10, no. 12, pp. 833-834, 2010.

[2] A. R. Tappuni, "Immune reconstitution inflammatory syndrome," Advances in dental research, vol. 23, no. 1, pp. 90-96, 2011.

[3] E. Letang, J. M. Miró, T. Nhampossa et al., "Incidence and predictors of immune reconstitution inflammatory syndrome in a rural area of Mozambique," PLOS ONE, vol. 6, no. 2, Article ID e16946, 2011.

[4] P. M. Grant, L. Komarow, J. Andersen et al., "Risk factor analyses for immune reconstitution inflammatory syndrome in a randomized study of early vs. deferred ART during an opportunistic infection," PLoS ONE, vol. 5, no. 7, Article ID el1416, 2010.

[5] D. M. Murdoch, W. D. F. Venter, C. Feldman, and A. Van Rie, "Incidence and risk factors for the immune reconstitution inflammatory syndrome in HIV patients in South Africa: A Prospective Study," AIDS, vol. 22, no. 5, pp. 601-610, 2008.

[6] I. Zanoni and F. Granucci, "Regulation and dysregulation of innate immunity by NFAT signaling downstream of pattern recognition receptors (PRRs)," European Journal of Immunology, vol. 42, no. 8, pp. 1924-1931, 2012.

[7] S. D. Lawn and M. A. French, "Immune reconstitution disease: recent developments and implications for antiretroviral treatment in resource-limited settings," Current Opinion in HIV and AIDS, vol. 2, no. 4, pp. 339-345, 2007.

[8] D. R. Boulware, K. H. Hullsiek, C. E. Puronen et al., "Higher levels of CRP, D-dimer, IL-6, and hyaluronic acid before initiation of antiretroviral therapy (ART) are associated with increased risk of AIDS or death," Journal of Infectious Diseases, vol. 203, no. 11, pp. 1637-1646, 2011.

[9] B. O. Porter, G. L. Ouedraogo, J. N. Hodge et al., "d-Dimer and CRP levels are elevated prior to antiretroviral treatment in patients who develop IRIS," Clinical Immunology, vol. 136, no. 1, pp. 42-50, 2010.

[10] S. A. Shelburne, F. Visnegarwala, J. Darcourt et al., "Incidence and risk factors for immune reconstitution inflammatory syndrome during highly active antiretroviral therapy," AIDS, vol. 19, no. 4, pp. 399-406, 2005. 
[11] D. R. Boulware, D. B. Meya, T. L. Bergemann et al., "Clinical features and serum biomarkers in HIV immune reconstitution inflammatory syndrome after cryptococcal meningitis: a prospective cohort study," PLoS Medicine, vol. 7, no. 12, Article ID e1000384, 2010.

[12] G. Martin-Blondel, L. T. Mars, and R. S. Liblau, "Pathogenesis of the immune reconstitution inflammatory syndrome in HIVinfected patients," Current Opinion in Infectious Diseases, vol. 25, no. 3, pp. 312-320, 2012.

[13] S. K. Sharma and M. Soneja, "HIV \& immune reconstitution inflammatory syndrome (IRIS)," Indian Journal of Medical Research, vol. 134, no. 12, pp. 866-877, 2011.

[14] J. P. B. Viola and A. Rao, "Molecular regulation of cytokine gene expression during the immune response," Journal of Clinical Immunology, vol. 19, no. 2, pp. 98-108, 1999.

[15] S. Feske, A. Rao, and P. G. Hogan, "The $\mathrm{Ca}^{2+}$-calcineurin-NFAT signalling pathway," New Comprehensive Biochemistry, vol. 41, pp. 365-401, 2007.

[16] C. Argyropoulos and A. Mouzaki, "Immunosuppressive drugs in HIV disease," Current Topics in Medicinal Chemistry, vol. 6, no. 16, pp. 1769-1789, 2006.

[17] R. Tadokera, G. Meintjes, K. H. Skolimowska et al., "Hypercytokinaemia accompanies HIV-tuberculosis immune reconstitution inflammatory syndrome," European Respiratory Journal, vol. 37, no. 5, pp. 1248-1259, 2011.

[18] M. E. Franks, G. R. Macpherson, and W. D. Figg, "Thalidomide," Lancet, vol. 363, no. 9423, pp. 1802-1811, 2004.

[19] Y. Xie, J. Sun, J. Su, L. Xu, Y. Huang, and B. Zhu, “Thalidomide for treatment of immune reconstitution inflammatory syndrome in a HIV patient with cutaneous mycobacterium avium complex infection," Journal of AIDS \& Clinical Research, vol. 6, article no. 530, 2015. 


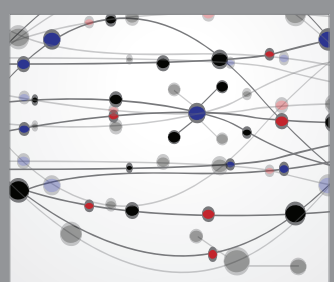

The Scientific World Journal
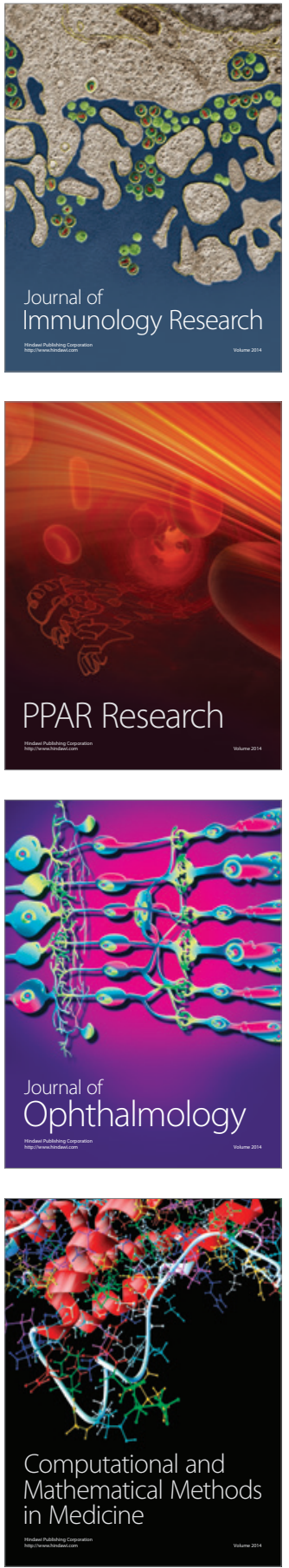

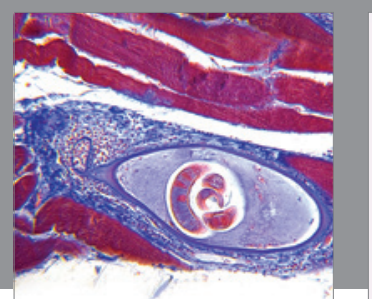

Gastroenterology Research and Practice
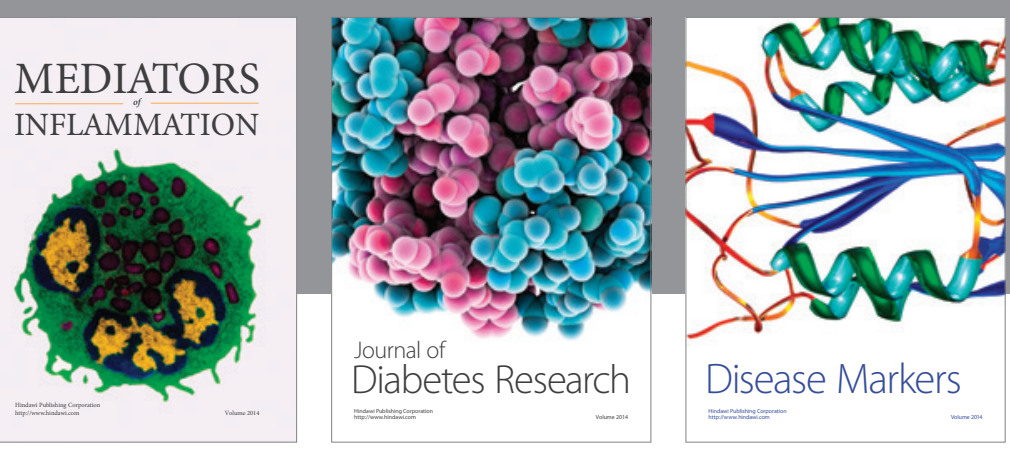

Disease Markers

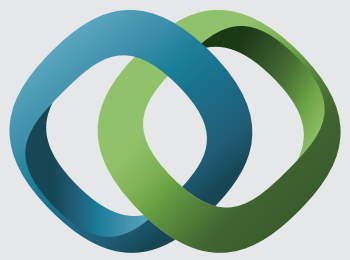

\section{Hindawi}

Submit your manuscripts at

https://www.hindawi.com
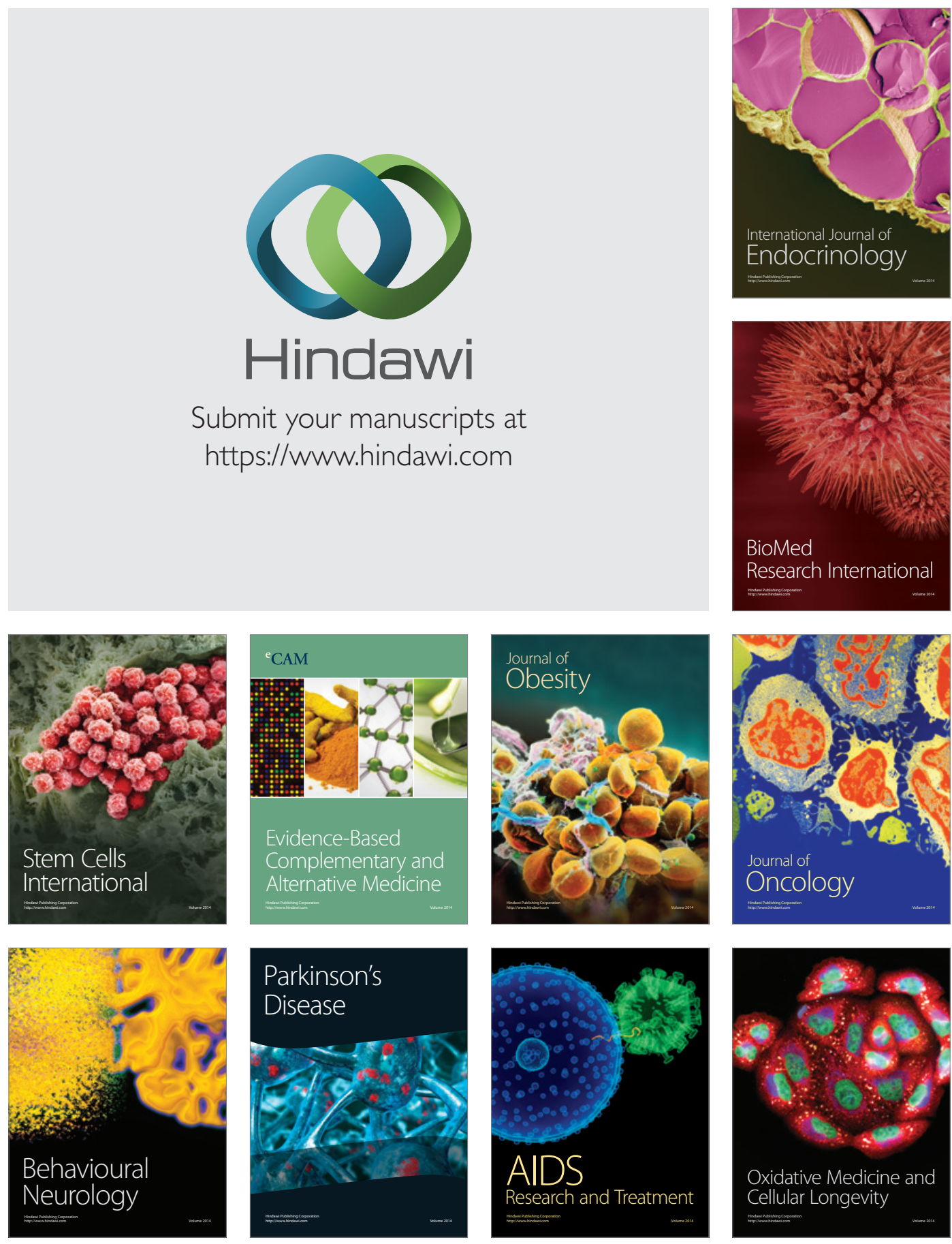